\title{
Design of Orthogonal UWB Pulse Waveform for Wireless Multi-Sensor Applications*
}

\author{
Hannu Olkkonen ${ }^{1}$, Juuso T. Olkkonen ${ }^{2}$ \\ ${ }^{1}$ Department of Physics and Mathematics, University of Eastern Finland, Kuopio, Finland \\ ${ }^{2}$ VTT Technical Research Centre of Finland, 02044 VTT, Finland \\ E-mail: hannu.olkkonen@uef.fi, juuso.olkkonen@vtt.fi \\ Received August 13, 2010; revised September 21, 2010; accepted October 28, 2010
}

\begin{abstract}
In this work we propose an orthogonal pulse waveform for wireless ultra wideband (UWB) transmission. The design is based on an ideal low-pass prototype filter having a windowed sinc impulse response. The frequency response of the prototype filter is transferred to the high frequency region using a specific sign modulator. The UWB pulse waveform comprises of the weighted summation of the left singular vectors of the impulse response matrix. The power spectral density of the pulse waveform fulfils the FCC constraint (allowed frequency band 3.1-10.6 GHz) for unlicensed UWB transmission. Applications of the UWB pulse waveform in multi-channel wireless sensor networks are considered.
\end{abstract}

Keywords: UWB, Wireless Sensor Networks

\section{Introduction}

The ultra wideband (UWB) technology is becoming a widely used wireless data transmission method in a variety of systems. The spectral density of the UWB pulses is restricted by the FCC constraint (allowed frequency band 3.1-10.6 GHz) for unlicensed UWB transmission [1]. Compared with the conventional impulse radio method, which uses the Gaussian monocycle pulses, this criterion brings new challenges to the design of the UWB pulse waveforms. In multi-channel wireless systems, the minimal crosstalk between the neighbouring channels is an essential requirement. One design method is based on the M-channel FIR filter bank and the UWB pulses are the impulse responses of the narrow band-pass FIR filters. The filter bank matching with the FCC criterion is designed with the Parks-MacClellan algorithm. A disadvantage of the FIR filter bank method is the slowly decaying impulse response, which prolongs the throughput rate of the transmission system. Recently, a different approach has been presented, where the UWB pulse $p(t)$ is constructed as a linear combination of the shifted orthogonal function $\phi(t)$ [2]

$$
p(t)=\sum_{k=0}^{p} B_{k} \phi(t-k)
$$

where $\phi(t)$ obeys the orthogonal constraint

*This work was supported by the National Technology Agency of Finland (TEKES).

$$
\int_{-\infty}^{\infty} \phi(t-k) \phi(t-l) d t=\left\{\begin{array}{l}
1 \text { for } k=l \\
0 \text { for } k \neq l
\end{array}\right.
$$

The information is coded to the $B_{k}$ sequence. The reconstruction is based on the correlation

$\left.\int_{-\infty}^{\infty} p(t) \phi(t-m) d t=\int_{-\infty}^{\infty} \sum_{k=0}^{p} B_{k} \phi(t-k)\right) \phi(t-m) d t=B_{m}$

where $m=0,1, \ldots, p$. The pulse generators and the correlators are usually analog circuits and $B_{k}$ is a binary sequence $B_{k} \in[0,1]$. A disadvantage of the correlation method is the long integration time required to obtain an adequate signal-to-noise ratio.

In this work, we study a method the UWB pulse waveform consists of the weighted summation of orthogonal vectors $\mathbf{u}_{\mathbf{k}}, k=1,2, \ldots, M$ having the dot product condition $\mathbf{u}_{\mathbf{k}}^{\mathrm{T}} \mathbf{u}_{\mathbf{l}}=1$ for $k=l$ and $\mathbf{u}_{\mathbf{k}}^{\mathrm{T}} \mathbf{u}_{\mathbf{l}}=0$ for $k \neq l$. In Section 2 we describe the design of the prototype filter, the UWB pulse waveform and the reconstruction method. Section 3 describes experimental results. In section 4 we discuss the application of the orthogonal UWB pulse waveform in wireless multi-sensor environments.

\section{Theoretical Considerations}

\subsection{Filter Design}

The prototype filter consists of the ideal low-pass filter, which has the impulse response 


$$
h(t)=A \frac{\sin \left(\omega_{c} t\right)}{\omega_{c} t}=A \operatorname{sinc}\left(\omega_{c} t\right)
$$

where $\omega_{c}$ is the cut-off frequency. The frequency response

$$
H(j \omega)=\left\{\begin{array}{l}
A \text { for }|\omega| \leq \omega_{c} \\
0 \text { for }|\omega|>\omega_{c}
\end{array}\right.
$$

The discrete-time is defined as $t_{n}=n T, n \in \mathbb{N}$ and the discrete impulse response of the low-pass filter as $\hat{h}_{n}=A \operatorname{sinc}\left(\omega_{c} n T\right)$.The impulse response should be symmetrical and compactly supported. The compact support is achieved by weighting the discrete impulse response $h_{n}$ by a window $w_{n}$, which eliminates the side lobes due to the truncated impulse response. We have for $\hat{h}_{n}=A w_{n} \operatorname{sinc}\left(\omega_{c} n T\right) n=-N, \ldots, N$. The design of the window functions (Hanning, Hamming, Blackman etc.) is well described in signal processing literature [3]. We applied the cosine window

$$
w_{n}=\alpha-(1-\alpha) \cos [\pi(n+N) / N], n=-N, \ldots, N
$$

where $\alpha=0.5$ for Hanning window and $\alpha=0.54$ for Hamming window.

The power spectral density of the prototype filter is concentrated on the frequency range $\left[0, \omega_{c}\right]$. To validate the FCC constraint (allowed band $3.1-10.6 \mathrm{GHz}$ ) for unlicensed UWB transmission [1] the key idea in this work is that the discrete impulse response of the lowpass filter is sign modulated as

$$
h_{n}=(-1)^{n} \hat{h}_{n}
$$

In continuous-time domain this corresponds to the modulation of the signal by $\cos (\pi t)$. The sign modulator has been used in VLSI wavelet transform chips to simplify the construction of the analysis and synthesis filters [4]. In $\mathrm{z}$ transform domain this equals to the replacement of the $\mathrm{z}$ variable by $-\mathrm{z}$. In frequency domain the frequency spectrum becomes a mirror image with respect to $\omega=\pi / 2$ (half of the Nyquist frequency). The original low-pass filter has the frequency components up to the cut-off frequency. The sign modulated impulse response has the mirror image spectrum, which is concentrated in the range $\omega_{\min }-\pi$. The upper limit frequency is defined by the Nyquist frequency, which should be selected under $10.6 \mathrm{GHz}$. Then the cut-off frequency $\omega_{c}$ of the low-pass filter so that the lowest frequency component of the sign modulated sequence matches to $3.1 \mathrm{GHz}$.

\subsection{UWB Pulse Waveform}

The impulse response matrix is defined as

$$
\mathbf{H}=\left[\begin{array}{cccc}
h_{-N} & h_{-N+1} & \cdots & h_{0} \\
h_{-N+1} & h_{-N+2} & \cdots & h_{1} \\
\vdots & \vdots & \vdots & \vdots \\
h_{0} & h_{1} & \cdots & h_{N}
\end{array}\right]
$$

where $h_{n}, n=-N, \ldots ., N$ is the impulse response of the high-pass filter. The output pulse vector

$\mathbf{p}_{n}=\left[p_{n} p_{n+1} \ldots p_{n+N}\right]^{T}$ of the UWB pulse generator is described by the state-space equation

$$
\begin{aligned}
\mathbf{x}_{n+1} & =\mathbf{A} \mathbf{x}_{n} \\
\mathbf{p}_{n} & =\mathbf{H} \mathbf{x}_{n}
\end{aligned}
$$

where the state vector $\mathbf{x}_{n} \in R^{(N+1) \times 1}$, the state transition matrix $\mathbf{A} \in R^{(N+1) x(N+1)}$. The usual problem is to solve the state transition matrix for the known signal. We seek the solution for the inverse problem, where the impulse response matrix $\mathbf{H}$ is known but the state vector must obey specific constraints.

By the singular value decomposition (SVD) of the impulse response matrix we have $\mathbf{H}=\mathbf{U} \boldsymbol{\Sigma} \mathbf{V}^{\mathbf{T}}$, where matrices $\mathbf{U}=\left[\begin{array}{llll}\mathbf{u}_{1} & \mathbf{u}_{2} & \ldots \mathbf{u}_{\mathrm{N}+1}\end{array}\right]$ and $\mathbf{V}=\left[\begin{array}{llll}\mathbf{v}_{1} & \mathbf{v}_{2} & \ldots & \mathbf{v}_{\mathrm{N}+1}\end{array}\right]$ contain the left and right singular vectors (column vectors) of the matrix $\mathbf{H}$ and matrix

$\boldsymbol{\Sigma}=\operatorname{diag}\left\{\sigma_{1} \sigma_{2} \ldots \sigma_{N+1}\right\}$ the singular values. Matrices $\mathbf{U}$ and $\mathbf{V}$ are unitary, i.e. $\mathbf{U}^{-1}=\mathbf{U}^{\mathbf{T}}$ and $\mathbf{V}^{-1}=\mathbf{V}^{\mathbf{T}}$. The eigenvectors are orthogonal, i.e.

$$
\mathbf{u}_{\mathbf{k}}^{\mathrm{T}} \mathbf{u}_{\mathbf{l}}=\mathbf{v}_{\mathbf{k}}^{\mathrm{T}} \mathbf{v}_{\mathbf{l}}=\left\{\begin{array}{l}
1 \text { for } k=l \\
0 \text { for } k \neq l
\end{array}\right.
$$

We may deduce

$$
\begin{aligned}
\mathbf{H V} & =\mathbf{U} \boldsymbol{\Sigma} \Rightarrow \mathbf{H}\left[\begin{array}{lll}
\mathbf{v}_{1} & \mathbf{v}_{2} & \ldots \mathbf{v}_{\mathbf{N}+1}
\end{array}\right] \\
& =\left[\begin{array}{llll}
\sigma_{1} \mathbf{u}_{1} & \sigma_{2} \mathbf{u}_{2} \ldots \sigma_{N+1} \mathbf{u}_{\mathbf{N}+1}
\end{array}\right] \Rightarrow \mathbf{H v}_{\mathbf{k}}=\sigma_{k} \mathbf{u}_{\mathbf{k}}
\end{aligned}
$$

for $k=1,2, \ldots, N+1$. The state vector $\mathbf{x}_{n}$ can be represented by a linear combination of the right singular vector basis as

$$
\mathbf{x}_{\mathbf{n}}=\alpha_{1} \mathbf{v}_{\mathbf{1}}+\alpha_{2} \mathbf{v}_{\mathbf{2}}+\ldots+\alpha_{N+1} \mathbf{v}_{\mathbf{N}+\mathbf{1}}
$$

where $\alpha_{k}(k=1,2, \ldots, N+1)$ are scalar coefficients. Now the UBW pulse vector can be written as

$$
\begin{aligned}
\mathbf{p}_{\mathbf{n}} & =\mathbf{H} \mathbf{x}_{\mathbf{n}}=\alpha_{1} \sigma_{1} \mathbf{u}_{\mathbf{1}}+\alpha_{2} \sigma_{2} \mathbf{u}_{\mathbf{2}}+\ldots+\alpha_{N+1} \sigma_{N+1} \mathbf{u}_{\mathbf{N}+\mathbf{1}} \\
& =\sum_{k=1}^{N+1} \alpha_{k} \sigma_{k} \mathbf{u}_{k}=\sum_{k=1}^{N+1} w_{k} \mathbf{u}_{k}
\end{aligned}
$$

where $w_{k}=\alpha_{k} \sigma_{k}$. Equation (13) shows that the UWB pulse vector is constructed from a weighted summation of the left singular vectors.

The trade-off in the present method is that several singular values in the singular value decomposition of the impulse response matrix $\mathbf{H}$ are very low. As an example, if we select $N=32$ and $\omega_{c}=0.6 \pi, 13$ of the singular values are tiny. The corresponding left singular vectors are not appropriate for the UWB pulse waveforms. However, 20 of the 33 singular values are at the adequate level. The invalid eigenvectors can be rejected from the summation by defining their weighting coefficients $w_{k}=0$ for $k=M+1, \ldots, N+1$. The weighting sequence carries the information, which can be re- 
constructed via the orthogonal condition

$$
\mathbf{p}_{\mathbf{n}}^{\mathbf{T}} \mathbf{u}_{\mathbf{l}}=\sum_{k=1}^{M} w_{k} \mathbf{u}_{\mathbf{k}}^{\mathbf{T}} \mathbf{u}_{\mathbf{l}}=w_{l} ; l=1,2, \ldots, M
$$

For binary weighting sequence $w_{k} \in[-1,+1]$ one UWB pulse waveform may involve $M$ bits of information.

\section{Results}

A numerical study was carried out on the effect of window on the Fourier spectrum of the UWB pulse waveforms. Compared with the rectangular window the cosine window had a significant improvement in the stop-band. The $\alpha$ parameter was varied between $0.50, \ldots, 0.54$. The best attenuation in the stop-band was obtained at $\alpha=0.54$. The number of valid singular vectors was not dependent on the $\alpha$ parameter. The stop band (0-3.1 $\mathrm{GHz}$ ) attenuation was typically 170-180 dB surpassing clearly the FCC criteria [1].

The UWB pulses were synthesized by a programmable $\mathrm{RF}$ pulse generator containing a 12 bit DAC and a RF tranceiver unit. The information coded to the UWB pulse was a 12 bit word $w_{k} \in[-1,+1] k=1,2, \ldots, 16$. The measurements were carried out using a high-speed memory oscilloscope equipped with an RF antenna probe. In practical measurements, noise arising in electronic circuits interferes with the results. Figure 1 describes the transmitted and received UWB pulse waveforms in the presence of low noise interference. Figure 2 illustrates the reconstructed 16-bit word. In Figure 3 the measurement was repeated in heavy noise environment. The peak -to-peak noise level was about $50 \%$ of the peak-to-peak value of the transmitted UWB pulse waveform. The reconstructed 16-bit word is still perfectly recovered (Figure 4).

\section{Discussion}

The distinct advantage of the use of the orthogonal UWB pulse vectors compared with the correlation methods is involved in their compact support enabling reconstruction via dot products.

The power spectral density of the UWB pulses clearly overrides the FCC criteria. In previous works (see e.g. [5]) the prolate spheroidal functions have been applied to design the orthogonal eigenvectors, which are used as UWB pulses. Our method based on the state-space modelling of the UWB pulse generator yields UWB pulse waveforms, which comprise of the weighted summation of the left singular vectors of the impulse response matrix. The weighting sequence carries out the transmitted information. Compared with the Gaussian monocycle pulse, which carries only one bit of information, the
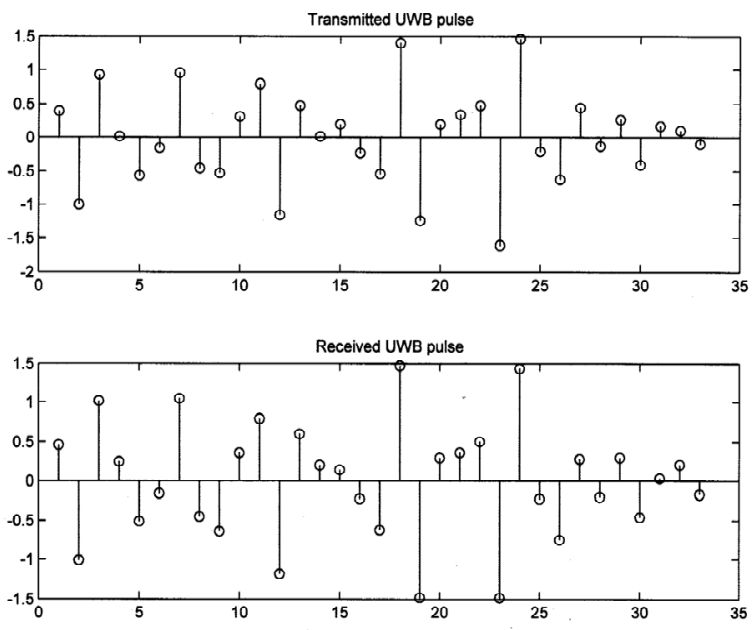

Figure 1. The transmitted and received UWB pulse waveforms in low noise environment.

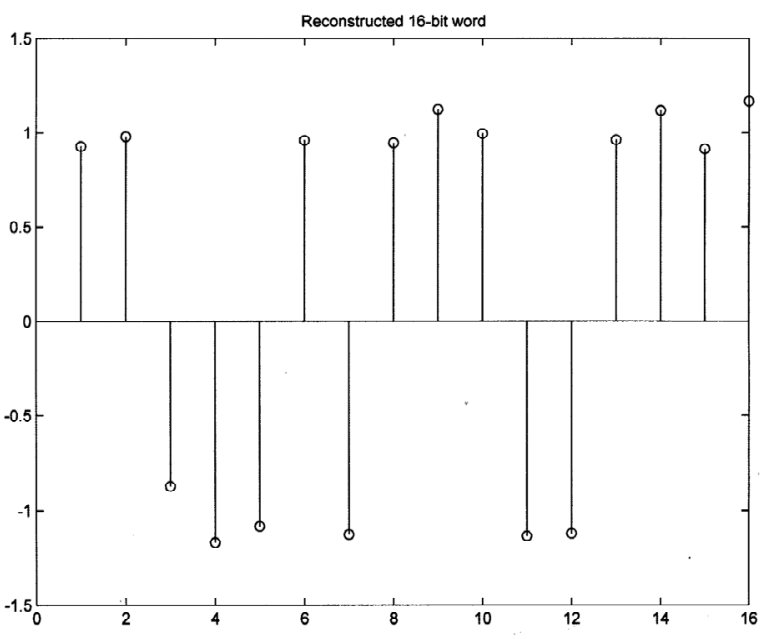

Figure 2. The reconstructed 16-bit word.
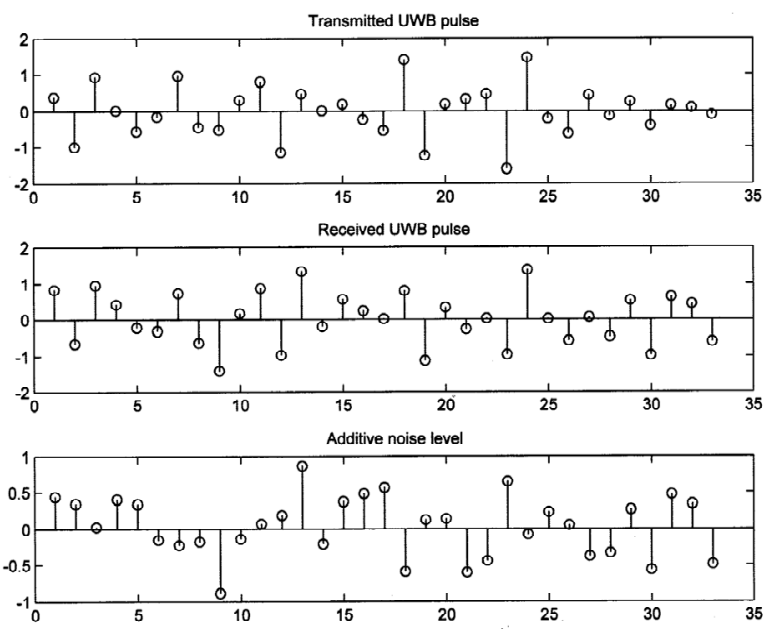

Figure 3. The UWB pulse waveform measured in heavy noise environment. 

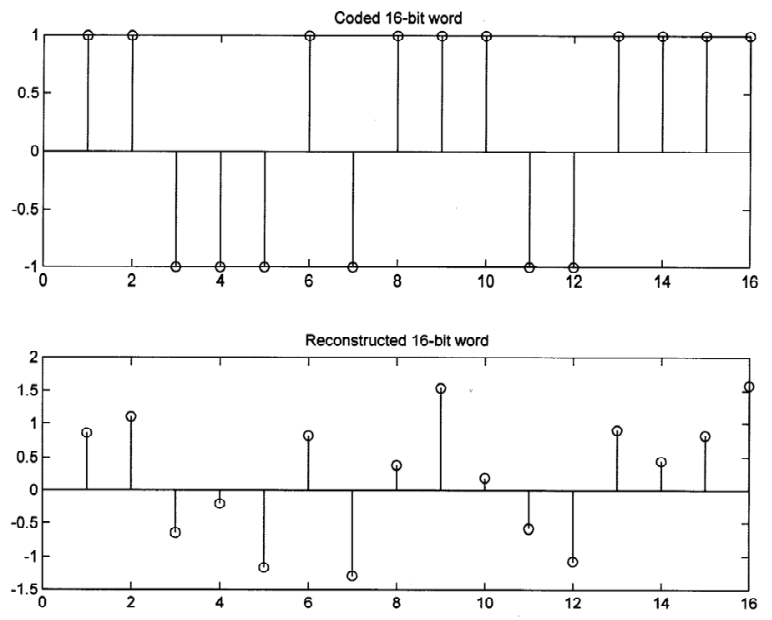

Figure 4. The coded and reconstructed 16-bit word.

adaptation of the UWB pulse waveform increases the bit rate by a factor of $M$ (14). In this work we used a binary 16-bit word as coded information. Our experiments have warranted that the reconstruction succeeds in heavy noise environment (Figures 3 and 4). On the other hand, the radiation waste is reduced in the 1/16 ratio.

In many wireless sensor applications the RF circuits with digital logic would be preferable to analog circuits. The construction of the UWB pulse generator can be made in VLSI environment [6]. The coding of the binary information needs only an adder and the register bank for left singular vectors. The output of the adder circuit can be directly interfaced to the DAC and the RF transmitter unit. The transmitted 16-bit word can comprise of the device address, information bits and error detection bits. This simplifies the design of the wireless multi-sensor measurements compared with one bit per pulse transmission systems using impulse streams [7-10].

\section{Acknowledgements}

We are indebted to the reviewers' comments, which improved the manuscript significantly.

\section{References}

[1] Y. P. Nakache and A. F. Molisch, "Spectral Shaping of Uwb Signals for Time-Hopping Impulse Radio,” IEEE Journal of Selected Areas of Communications, Vol. 24, No. 4, 2006, pp. 738-744.

[2] M. Wang, S. Yang and S. Wu, "A GA-based UWB Pulse Waveform Design Method,” Digital Signal Processing, Vol. 18, No. 1, 2008, pp. 65-74.

[3] A. V. Oppenheim and R. W. Schafer, "Discrete-Time Signal Processing,” Englewood Cliffs: Prentice-Hall, New Jersey, 1989.

[4] H. Olkkonen and J. T. Olkkonen, "Simplified Biorthogonal Discrete Wavelet Transform for VLSI Architecture Design,” Signal, Image and Video Processing, Vol. 2, No.2, 2008, pp. 101-105.

[5] B. Parr, B. Cho, K. Wallace and Z. Ding, "A Novel Ultra-Wideband Pulse Design Algorithm,” IEEE Communications Letters, Vol. 7, No. 5, 2003, pp. 219-221.

[6] M. Miao and C. Nguyen, "On the Development of an Integrated CMOS-Based UWB Tunable-Pulse Transmit Module," IEEE Transactions on Microwave Theory and Techniques, Vol. 54, No. 10, 2006, pp. 3681-3687.

[7] I. Maravic and M. Vetterli, "Sampling and Reconstruction of Signals with Finite Rate of Innovation in the Presence of Noise," IEEE Transactions on Signal Process, Vol. 53, No. 8, 2005, pp. 2788-2805.

[8] P. L. Dragotti, M. Vetterli and T. Blu, "Sampling Moments and Reconstructing Signals of Finite Rate of Innovation: Shannon Meets Strang-Fix,” IEEE Transactions on Signal Process, Vol. 55, No. 5, 2007 pp. 1741-1757.

[9] I. Jovanovic and B. Beferull-Lozano, "Oversampled A/D Conversion and Error-Rate Dependence of Nonband Limited Signals with Finite Rate of Innovation," IEEE Transactions on Signal Process, Vol. 54, No. 6, 2006, pp. 2140-2154.

[10] J. T. Olkkonen and H. Olkkonen, "Reconstruction of Wireless UWB Pulses by Exponential Sampling Filter," Wireless Sensor Network, Vol. 2, No. 6, 2010, pp. 411491. 